\title{
DESEMPEÑOS DE GÉNERO, SEXO Y RAZA EN EL MUSEO
}

\section{PERFORMANCE OF GENDER, SEX AND RACE IN THE MUSEUM}

\author{
Mar García Ranedo \\ Universidad de Sevilla
}

\section{RESUMEN}

Esta investigación aborda y subraya cómo el museo en el ámbito de las subjetividades relativas al género, sexo y raza ha sido regresivo y particularista. Regresivo por el reiterado regreso a las narrativas represoras de la historia del arte y particularista por la insuficiencia de un repertorio representacional y crítico que afronte posiciones feministas o consideradas de alteridad. A través de la proyección de la obra videográfica titulada $E$ l señuelo -de 12 minutos de duración- haré un recorrido conceptual y analítico por algunos museos del siglo XX y XXI, considerados escaparates internacionales del arte contemporáneo.

Palabras clave: Museo, género, subjetividad, feminista, raza, alteridad.

\section{ABSTRACT}

This proposal deals with how the museum has been regressive and particularistic in the field of subjectivities related to gender, sex and race. Regressive for the repeated return to the repressive narratives of the history of art and particularist for the incompleteness of the representational and critical repertoire that addresses feminist or considered positions of otherness. Through the projection of a video work entitled The decoy -12 minutes long- I make a conceptual and analytical journey through some 20th and 21 st century museums considered international showcases of contemporary art.

Keywords: Museum, Gender, Subjectivity, Feminist, Race, Otherness.

\section{SUMARIO}

1. Memoria, verdad y compromiso. 2. Alteridad, marginalidad y exclusión. 3. Recorrer el museo. 4. El señuelo. 5. Referencias y bibliografía. 


\section{Memoria, verdad y compromiso}

Este escrito traza un recorrido analítico por el museo. Resulta difícil entender los vacíos en la historia del arte en lo relativo al feminismo. Recuerdo, cuando era estudiante, mis visitas a los museos. Entonces no me cuestionaba esa omisión, pero si me hubieran preguntado por exposiciones individuales que incluyeran a artistas mujeres sólo hubiera podido mencionar unas pocas. Sin duda, hoy se ha avanzado mucho en la presencia en los museos no únicamente de la mujer sino de ideologías y soportes creativos asociados a ésta y antes devaluados. Aun así, las intervenciones feministas en la escena artística vivieron en la marginalidad cultural la mayor parte del siglo XX. Este escrito propone una revisión de momentos puntuales del siglo pasado en relación a la ocupación del museo por parte del arte feminista, de tal forma que el hecho de la no-ocupación nos revele estructuras donde a la mujer se la invisibiliza como artista, más allá de la consideración como desnudo femenino o representación idealizada para el disfrute de una visualidad masculina heterosexual que actúa como inconsciente de la sociedad patriarcal (Mulvey, 2001). En 1989, las Guerrilla Girls nos advertían que en la sección dedicada al arte moderno del Metropolitan Museum solamente el $5 \%$ eran mujeres, a pesar de que el $85 \%$ eran desnudos femeninos.

Es a partir de las décadas de los sesenta y setenta del siglo pasado cuando la escena cultural norteamericana resulta especialmente combativa por parte de un grupo de mujeres que reclamaron decididamente un lugar en la historia. Ese «levantamiento» exigía el compromiso de las creadoras, que tendían a ser concebidas como un colectivo homogéneo de mujeres demarcadas por objetivos comunes y miradas semejantes. En los años sesenta el «colectivo» toma conciencia de cómo la protesta es un mecanismo eficaz con el que combatir públicamente las estructuras políticas que gobiernan, ordenando sus vidas y truncando sus sueños y aspiraciones. En el contexto norteamericano se crea, en 1966, la Organización Nacional de las Mujeres con la intención de legitimar política y simbólicamente un movimiento de liberación capaz de sostener ideológicamente la protesta contra la opresión, la marginación y las demandas para un cambio social y político. Las artistas feministas retomaron los principios que articulaban el movimiento de liberación de las mujeres como argumento para trabajar desde el arte. De este modo, demandaron la falta de inclusión de artistas mujeres en las galerías y los museos, al mismo tiempo que buscaron recuperar aquellos lenguajes entendidos como «arte menor» por proceder del ámbito de la artesanía y de las artes decorativas. La visión de unidad que aparentaba dicha revuelta fue modificándose en décadas posteriores con la influencia, en los ochenta, del postestructuralismo, el psicoanálisis, los estudios culturales y poscoloniales. Así, el discurso feminista en el ámbito del arte se 
fragmentó en múltiples derivas o feminismos, postfeminismos y transfeminismos difíciles de teorizar, definir y concretar por tratarse de identidades no-binarias, cruzadas, y complejas para limitarlas y determinarlas en categorías (Solá, 2013:15).

Los discursos relativos a la mujer se analizan, por tanto, desde cuestiones concernientes a la raza, el género, la diferencia sexual, la orientación sexual, las teorías queer, etc. Definir el término "feminismo» es difícil, sería ajustarlo o delimitarlo a una única explicación o interpretación en la que todas aquellas personas que reclaman una identidad feminista logren verse reflejadas; si bien, en el actual caleidoscopio que conforman los diferentes feminismos, algunas ideas pueden ser consideradas comunes. En este sentido, recordemos la definición audaz y amplia que Elena Reckitt ofrece sobre arte feminista: «el feminismo es la convicción de que el género ha sido, y sigue siendo, una categoría fundamental para la organización de la cultura. Pero el patrón de esa organización generalmente favorece a los hombres sobre las mujeres»' (Reckitt, 2001: 18). O la aportada por la comisaria Lucy Lippard (1980): «El arte feminista es un sistema de valores, una forma de vida, una estrategia de cambio».

La historia del arte moderno ha estado empeñada en analizar la producción artística no como artefactos culturales activados desde códigos que enlazan con la realidad social y política, sino como objetos que hay que observar, desde dentro del propio marco de representación, conectados con el pasado. En este sentido, diremos que la crítica feminista ha sido expansiva; ha ampliado los límites normativos cuestionando todo lo que, en el ámbito cultural, ocurre fuera de lo enmarcado y establecido en el orden patriarcal para ofrecer otras conexiones capaces de interpretar las obras de arte de las mujeres como inscripciones de la memoria y el tiempo desde la diferencia sexual. Diríamos que existe un tipo de revisionismo crítico que no se ajusta a los convencionalismos de los relatos dominantes de la historia del arte, así como un modo de escritura crítica y de producción artística que se resuelven desde esas fronteras cruzadas; desplazándose entre lo enunciado y lo ocluido o entre lo visto y lo oculto.

El legado de las primeras artistas feministas es clave para entender cómo la historia cultural de las mujeres ha sido obviada y excluida del relato oficial, heteronormativo y prejuiciado, focalizado en un centrismo de raza y género y la prevalencia de lo euroamericano y blanco que describe el escenario del arte del siglo XX. La inclusión en los programas expositivos de los museos de artistas mujeres de color, coincide con estrategias políticas ocupadas en satisfacer una demanda social centrada en representar la diversidad cultural. En 1971, en un artículo publicado en Art News, la profesora norteamericana Linda Nochlin

1 Traducido por la autora del artículo del texto original en inglés: «Feminsm is the convition that gender has been, and continues to be, a fundamental category for the organization of culture. Moreover, the pattern of that organization usually favours men over women». 
(1971) formuló una pregunta clave: «Why have there been no great women artists?»². Por qué la historia de los grandes hombres artistas no tiene como correlato a mujeres en la historiografía canónica.

Los programas institucionales de arte feminista que Judy Chicago en 1970 llevó a cabo en el Fresno State College, California, y más tarde en 1971 junto a Miriam Schapiro en el Cal Arts en Valencia, California, fueron especialmente importantes a la hora de generar un contexto en el que empoderar a las mujeres artistas concienciándolas de la importancia de llevar a cabo un trabajo artístico basado en la experiencia personal. Un año más tarde, en 1972, se creó la Womanhouse, un espacio expositivo en el que las reglas del arte de las corrientes dominantes quedaban volteadas por veinticuatro artistas mujeres al entender dicho espacio como una prolongación pública de lo doméstico y privado. El espacio como contenedor del objeto artístico comenzaba, de esta forma, a transfigurar sus normotipos (entendido en su doble acepción puesto que incorporaba nuevos figurantes al territorio de la representatividad).

El museo contenedor y legitimador de las prácticas artísticas ha sido excluyente y segregacionista en relación a los feminismos. El museo moderno ha creado notables diferencias en los distintos ámbitos del conocimiento mediante la clasificación y preservación de objetos de arte que han resistido el paso del tiempo estableciéndose como identidad artística e histórica de una cultura. Al mismo tiempo, sin embargo, ha negado u omitido otras obras de contenido no ya inaceptable sino inapreciable para la conveniencia del trazado lineal y hegemónico del discurso museal. Las minorías, el género o cualquier tipo de inadecuación al librepensamiento y las energías de mercadotecnia neoliberales se han trazado desde estructuras de difusión «menores» alejadas, en todo momento, de las grandes construcciones icónicas. Ni siquiera algunos ismos o prácticas artísticas que promovían el alejamiento del museo y la instauración del hecho y objeto artísticos como el land art fueron ajenos al rito cultual posterior y su inserción en los espacios institucionales del poder: sólo hay que observar los recortes deconstructivos o decollages de Gordon Matta Clark en la colección del MoMA. Esta aparente aleatoriedad es, por el contrario, deliberada; es, con toda certeza, una estrategia intencionada ya que lo descartado responde a un patrón feminista, quedando la mujer privada del orden y reglamento creativos propios a la institución.

The Dinner Party de Judy Chicago fue mostrada al público por primera vez en el Museo de Arte Moderno de San Francisco, en 1979, activando un debate que ha durado décadas. Discusión que, a día de hoy, perdura acerca de la sexualidad y el desafío al 
patriarcado, propiciado por las treinta y nueve mujeres representadas (mediante platos de cerámica con formas de vulva) elegidas por sus contribuciones relevantes al ámbito de las humanidades y la ciencia. Lo que Judy Chicago consiguió fundamentalmente fue remover las conciencias. Como impulsora del movimiento de arte feminista de la costa oeste de Estados Unidos se centró en visibilizar la exclusión de las artistas de California en las exposiciones organizadas desde el ámbito institucional, al mismo tiempo que ponía de manifiesto cómo han sido continuamente silenciadas en publicaciones y revistas de arte especializadas.

El 26 de julio de 1990, The Dinner Party fue cuestionada, desde argumentos que aludían a la ética, en la Cámara del Congreso de los Estados Unidos de América por los políticos, todos ellos hombres. Durante una hora y veintisiete minutos -tiempo que duró la controversia- se debatió y deliberó lo inapropiado e inadecuado de The Dinner Party como obra para el ámbito museístico. El político republicano del Congreso norteamericano Robert K. Dornan señaló: "The Dinner Party no es arte, es pornografía»; el también republicano Dana Rohrabacher lo denominó «arte sexual raro» ${ }^{3}$. Finalmente, de los 124 miembros del congreso 123 votaron de forma negativa y hubo una abstención, para que la enmienda se aceptara y The Dinner Party no pudiera ser mostrada en el museo.

Tras la estadía de The Dinner Party en el San Francisco Museum of Modern Art y hasta su llegada definitiva al Brooklyn Museum en 1981, la pieza viajó por seis países y fue expuesta en catorce centros de arte. A pesar del interés, medido por el número de visitas recibidas a los largo de esos años de itinerancia, también fue rechazada por algunos museos: la Memorial Gallery de la Universidad de Rochester y el Seattle Art Museum. Ambas instituciones alegaron la dificultad que suponía su transporte e instalación. Aunque es obvio que estos inconvenientes pueden entorpecer su exhibición, lo cierto es que Charles Cowles, por aquel entonces comisario de arte del Seattle Art Museum, manifestó en el periódico Seattle Sun: «No considero la obra buen arte sino un proyecto interesante de un grupo de mujeres cuyo líder es una artista». Remarcó su alegato diciendo: "No mostramos declaraciones políticas feministas» (Jane, 2013: 185-187). The National Collection of Fine Arts y el Smithsonian así como el Institute for the Arts de la Universidad de Rice también rechazaron exponer The Dinner Party. Sus portavoces adujeron la no coincidencia de la obra con los objetivos conceptuales de sus programas. La instalación de Judy Chicago, con las treinta y nueve representaciones simbólicas de los genitales femeninos, resultó controvertida al mismo tiempo que incómoda para la institución y la crítica conservadoras, atrayendo a entusiastas y a detractores. La teórica y comisaria de arte feminista Amelia Jones se ocupó

3 https://Women.art.revolution.limited.2010 
de rebatir cualquier juicio negativo que buscara clasificar la pieza tan sólo como una obra de arte moderna. Apuntó que su complejidad e interés radica en lo que revela en relación a la evolución y desarrollo del feminismo y el arte: «subvierte descaradamente los sistemas de valores modernistas que privilegian el puro objeto estético sobre el sentimentalismo degradado de las artes domésticas y populares» ${ }^{4}$ (Jones, 1996: 87). Sin embargo, antes de su exhibición el 18 de octubre de 1980 en el Brooklyn Museum, el ensayista y crítico de arte del New York Times, Hilton Kramer (1980), afirmó con rotundidad que The Dinner Party era «arte muy malo». En cualquier caso, para las feministas, el derecho al empleo del cuerpo era un modo de establecer dominio sobre su propia naturaleza y derribar tabúes en torno al miedo y la fobia a la representación de órganos sexuales femeninos.

The Dinner Party (comprada por la filántropa Elizabeth A. Sackler en 2001 con la intención de donarla al museol fue la primera obra que formó parte de un área dedicada exclusivamente al arte feminista dentro del espacio institucional, en el Brooklyn Museum. En 2002 se mostró al público como instalación permanente. Este hecho motivó a la dirección del museo la ideación de un centro de arte feminista que contribuyera a la investigación al mismo tiempo que educara y concienciara al público de la importancia de este arte como legado histórico. Incluir el arte feminista en el museo es, por otra parte, aproximarlo al canon como modelo y centrar el análisis de las obras en cuestiones relativas a la calidad y la genialidad. La conceptualización de un programa de arte feminista en el marco de la institución es, a fin de cuentas, un modo de reducir la «problemática feminista» a una cuestión de definición o de demarcación de lo que es arte feminista o no; o qué es buen arte feminista o mal arte. En 1977, la artista Mary Kelly, en una conferencia titulada Arte y política sexual, señaló como problemático, desactivar «lo ideológico» ${ }^{5}$ del concepto «prácticas feministas» al enmarcarlas en la institución (Kelly, 2001). Por su parte, Griselda Pollock subraya que «el proceso discursivo de mistificación puede incluir tanto a los artistas héroes del high modernism, como a las mujeres artistas que también han acabado siendo mistificadas como heroínas del feminismo» (Pollock, 2010: 26):

Aprender arte a través del discurso canónico, es conocer la masculinidad como poder y significado, y los tres como idénticos con la Verdad y la Belleza. Mientras el feminismo también intente ser un discurso sobre el Arte, la Verdad y la Belleza, sólo puede confirmar la estructura

4 Amelia Jones en su ensayo La política sexual de The Dinner Party analiza las respuestas afirmativas y negativas y su significado global dentro del feminismo. La considera una obra compleja, con varias capas, desde las que se pueden abordar diferentes análisis. Jones señala que se trata de un intento de construir una coalición de mujeres para impugnar las exclusiones que justamente las han llevado a estar juntas.

5 Lo ideológico como un conjunto de prácticas sociales y formas de representación que tienen consecuencias políticas. Véase en Guasch, Ana María (2000) Los manifiestos del arte posmoderno, p. 329. 
del canon y al hacerlo corrobora la maestría y el poder masculinos, sean cual sean el número de nombres de mujeres que trate de añadir, o las narrativas históricas más completas que logre producir (Pollock, 2006: 9).

Al museo moderno le ha costado mucho esfuerzo trasladar las condiciones de la contemporaneidad en el arte. Se da un lapso sincrónico entre lo que se produce, debate e interesa dentro del arte y lo que el museo muestra. En relación al género, la raza y el sexo esa diacronía ha sido no sólo mantenida sino también alimentada desde la institución. Las posiciones críticas, las representaciones de desigualdad social, la diversidad racial y cuestiones relativas al sexo quedan emplazadas continuamente en un museo modernista que en la sociedad contemporánea está cada vez más ligado a los circuitos del capital y el entretenimiento. Esta sinergia lo configura como una mediación centrada en satisfacer las actuales políticas neoliberales que nos organizan, alejándose de cometidos indispensables para una re-interpretación de la historia centrada en revisiones críticas, el cuestionamiento de los modelos historicistas y la resistencia o la oposición al establecimiento canónico. El objetivo no es, por tanto, expandir los discursos hegemónicos establecidos, representados como canon, tampoco obviarlos sino contrarrestarlos. Es importante recuperar la memoria de las mujeres artistas, silenciada, excluida y desplazada a los márgenes, incluso como un «acto de supervivencia», en palabras de Adrienne Rich (citado en Pollock, 2010: 58). Al mismo tiempo, surge la necesidad de reconceptualizar la marginalidad de la producción cultural de las mujeres porque el compromiso con el pasado y la verdad es una forma de activismo político clave dado que «está en juego la supervivencia, la memoria, las subjetividades y las culturas» (Crimp, 2005: 7).

\section{Alteridad, marginalidad y exclusión}

A finales de la década de los ochenta, asistí a una conferencia de Douglas Crimp. Este teórico manifestó su posición crítica en relación a la necesidad de generar representaciones alternativas a los modelos dominantes canónicos desde la alteridad y la marginalidad social más allá del contexto del museo o la galería. La diferencia -lejos de implicar exclusión- debería ser entendida como un modo de producir significado y conocimiento, defendía Crimp. De hecho, su trabajo se centró en desarticular las corrientes homogeneizadoras, propias de la modernidad, que actúan como el canon heteronormativo, fetichizando la obra, transformándola en capital, dado que ese canon a veces era reproducido dentro de los propios movimientos de liberación, como el propio Crimp observó en la comunidad gay norteamericana con la 
crisis del sida. Si a Crimp se le señala como agitador cultural en defensa de los derechos de la comunidad homosexual refutando los prejuicios acerca del sida en colaboración con el grupo de activistas conocidos como ACT UP 6 , a Lucy Lippard se la considera una figura clave en el avance del arte feminista. Mujer polivalente, en muchos sentidos, no sólo como figura teórica o «trabajadora del arte», como a ella le gusta denominarse, sino como tenaz activista (fundó junto con un grupo de artistas un comité de mujeres artistas independiente, AWC), escritora literaria y de ficción, crítica de arte, comisaria, madre soltera, defensora de las artistas, feminista e investigadora. Lippard consideró que el arte conceptual ofrecía, a las mujeres artistas, una vía experimental desde la que expresar sus vivencias y situarse críticamente a través de la obra. La consideración de emplear el registro escrito acerca del acontecimiento cotidiano como estrategia conceptual de orden visual era un modo de generar experiencias artísticas alejadas del orden canónico masculinista; al mismo tiempo, también era entendido como una manera de reflexionar sobre los modos «desmaterializados» de producir arte en un sistema en el que prevalece el capital. El arte feminista, obviado del relato normativo, ajeno, por tanto, a las dinámicas de mercantilización de la obra y a las maniobras de legitimación de la institución museística encontraría en el arte conceptual la herramienta idónea desde la que mostrar resistencia al discurso normativo. Aunque estos pronunciamientos, un tanto utópicos en sus comienzos, acabarían absorbidos por el sistema del arte que los devolvería en forma de mercancía artística.

En cualquier caso, durante esa fase de emergencia del arte minimalista y conceptual en torno a 1968 y coincidiendo con el surgimiento de movimientos sociales de resistencia, como el movimiento por los derechos civiles y el movimiento de liberación de las mujeres, Lippard se politizó (Lippard, 2004: 7). Ella misma señala cómo a partir de 1970 cambió el rumbo de su vida marcada por el compromiso con el arte desde la óptica de mujer:

Me había retirado para escribir ficción, algo que había estado haciendo a lo largo de toda mi vida y que siempre he intentado que fuese mi profesión real. En primer lugar me di cuenta que el libro que planeaba escribir no venía de mí, sino de los artistas con los que vivía y sobre los que escribía. Ellos eran, por supuesto, en su mayoría hombres. Por trivial que pueda parecer visto en retrospectiva, fue como si la tierra se estremeciera, como una revelación... Cambió enteramente lo que yo quería hacer. Comencé a escribir para mí en lugar de para

6 Su primer ensayo acerca del sida, Sida: militancia y representación fue publicado dos años después de escribirlo en la revista October, $n^{\circ} 51$ y marcó el final de la relación entre la revista y el crítico. 
una audiencia de hombres imaginarios y, por extensión, comencé a escribir para las mujeres ${ }^{7}$ (Butler, 2012: 50).

Tras una década de activismo político desde el arte a favor de los derechos de las minorías y de posicionamiento feminista, la década de los ochenta supuso un retroceso en las políticas culturales, caracterizada por una vuelta al conservadurismo con la figura de Ronald Reagan en el gobierno americano y en el ámbito británico con Margaret Thatcher. Muchas de las propuestas, que durante la década anterior se habían consolidado, se vieron comprometidas por falta de fondos y ayudas económicas. De este modo, se frenaron aquellos proyectos expositivos feministas y aquellos espacios alternativos gestionados por mujeres artistas. La losa conservadora salpicaría incluso a los movimientos sociales de protesta, como el feminista, cuestionados por obviar todas las posibles y diversas subjetividades en relación a la identidad racial, de clase o sexual de la mujer, reduciendo el asunto a dos tipos: mujer blanca y mujer de color. Publicaciones como la antología feminista titulada This bridge called my back: writings by radical women of color, publicada en 1981, fueron muy influyentes en el contexto académico de los Estados Unidos para la teorización de los feminismos de raza, clase y sexualidad ${ }^{8}$. Se trataba de una antología que reunía escritos de mujeres de diversos orígenes en la que se dibujaban todas las posibles identidades de raza, etnia, género, sexo y condición sexual. El ensayo supuso un desafío a la idea de conceptualizar todos los feminismos como categoría única, al hecho de clasificar categorías identitarias vinculando sexo con género y al hábito de establecer diferencias jerárquicas en lugar de interrelaciones e interdependencias entre las diversas identidades y subjetividades.

El traslado al museo de todas estas circunstancias identitarias relativas a la mujer supondría considerar que dicho lugar es capaz de ofrecerse como un territorio dialéctico comprometido. Por tanto, supondría un espacio que se posiciona críticamente para contener todas las posibles interpretaciones de la realidad cultural y todas las posibles subjetividades que conviven en este mundo, desde lo racial hasta lo afectivo. Sin embargo, el museo se ha distinguido por ser conformador de formas selectivas de pensamiento y memoria. El

7 Traducido por la autora del artículo del texto original en inglés: «l had retreated to write fiction, something I had been doing in fits and starts all my life and have always intented to make my real career.

First, I realised that the book I planned to write didn't come from me, but from artists I lived with and wrote about. They were, of course, mostly men. And second, I realised that I was ashamed of being a woman. Trite as this may sound in retrospect, it came as an earth-shaking revelation... It changed entirely what I wanted to do. I began to write for myself rather than for some imaginary male audience and, by extension, I began to write for women».

8 This bridge called my back: writings by radical women of color es una antología feminista publicada por primera vez en 1981 por Persephone Press, editado por los antropólogos Cherríe Moraga y Gloria Anzaldúa. Posteriormente se han editado nuevas ediciones en 1983, 2008 y 2015. La última edición, 2015, ha sido publicada por State University of New York Press, Albany. La antología fue traducida al español bajo el título: Este puente, mi espalda: Voces de mujeres tercermundistas en los Estados Unidos. 
museo -en términos de emprendimiento- no ha desplazado a las mujeres artistas porque para desplazar necesitas previamente haber alcanzado o mantenido un espacio, cargo $\circ$ emplazamiento y la mujer históricamente nunca ha ocupado ese lugar de empoderamiento. La deslocalización de la mujer artista en el espacio museístico, de esta forma, no se genera por desplazamiento sino por aplazamiento. En este sentido, las historias de las mujeres artistas han sido construidas desde las narrativas propias de la modernidad. Martha Rosler, Adrian Piper, Rachel Rosenthal, Eleanor Antin, Marlene Raven, Nancy Spero, Silvia Sleigh, Sheila de Bretteville, Mirian Shapiro y Judy Chicago, entre otras, fueron interpretadas como colapso, arrostramiento y brote de un movimiento de representatividad ajeno a los modelos de participación museísticos o galerísticos, en cierto modo, concertados.

Lee Krasner (1908-1984) fue una pintora americana de origen judío vinculada al expresionismo abstracto. Se llamaba en realidad Leonora pero lo cambió por Lee para que su condición de mujer no la marginara en el mundo del arte, de tal forma que sólo firmaba con sus iniciales. Fue la esposa de Jackson Pollock y esta circunstancia predisponía a los críticos e historiadores de arte a interpretarla, al margen de su condición de mujer artista y compañera, como la distracción para el genio de su marido, dado que las grandes ideas requieren estar en soledad. Incluso, el reputado crítico de arte Arthur Danto comentó de Lee: «Es difícil verla salvo como una sombra de artistas más grandes que ella: su profesor Hofmann, su marido, y su brillante escuela de arte contemporáneo de New York». Tras la muerte de Pollock, periodistas especializados en arte contemporáneo le preguntaron a Lee, si sentía que había perdido su identidad al morir su esposo. Algunos críticos como Harold Rosenberg comenzaron a llamarla la «action widow» para reforzar la identidad del desaparecido Pollock como pintor de acción. Lee dedicó su vida a la pintura, sin embargo su primera exhibición en un espacio galerístico fue a la edad de 47 años. Tras la muerte de Pollock, la obra pictórica de Krasner comienza a despertar interés por la comunidad artística norteamericana (Girls, 1998: 86).

Otro ejemplo de artista silenciada es Sylvia Sleigh (Gales, 1916 - Nueva York, 2010). Fue una pintora figurativa en el escenario norteamericano en el momento en el que el expresionismo abstracto era objetivo de grandes exposiciones. A pesar de su precario reconocimiento, Sylvia se considera una figura clave en la escena feminista de la década de los setenta. Formó parte de la Women's Caucus of Art, de la cooperativa de mujeres artistas A.I.R. (Artists-in-Residence) y del grupo que fundó en 1973 la Soho Gallery, un espacio ideado y configurado para exponer a mujeres artistas entre 1974 y 1978. La obra de Sylvia comenzó a ser reconocida por la crítica especializada poco antes de su muerte, a partir de la exposición celebrada en el año 2007, organizada por el MoCA de Los Ángeles, bajo el título de WACK! Art and the Feminist Revolution. 
Algo parecido le ocurrió a Nancy Spero (Cleveland, Ohio, 1926 - Nueva York, 2009). Como artista feminista y activista política, se señaló por pronunciarse contra el sistema patriarcal y los órdenes imperantes establecidos como modelos de la modernidad artística. Empleó la pintura para elaborar a través de la figuración, la poesía y la danza un entramado discursivo que situara la obra como protesta y oposición a la guerra de Vietnam al mismo tiempo que apoyó al movimiento de los derechos civiles. También buscó expresar y conjurar la alienación y marginalidad que sentía como artista mujer. Desechó emplear el lienzo para sus pinturas y dibujos y huir o alejarse con este gesto de la idea de autoridad y predominio que encarnaba el expresionismo abstracto y su nómina de pintores hombres. Formó parte de WAR (Women Artists in Revolution) y aunque en la década de los ochenta y los noventa su trabajo comienza a ser expuesto, no es hasta el 2007 cuando produce una instalación específica para una exposición internacional: la Bienal de Venecia, bajo el título Maypole: Taken No Prisoners. Con esta instalación, de nuevo, puso de manifiesto su rechazo a las políticas que se adoptan en los Estados Unidos en relación a los conflictos internacionales y la guerra. Aunque la primera exposición retrospectiva, tanto en Europa como en USA, fue celebrada en el año 2008, un año antes de su muerte, en el Museo Nacional Centro de Arte Reina Sofía.

\section{Recorrer el museo}

Como mediación, el museo es encargado de enmarcar, de visibilizar, en definitiva, de dar carácter oficial e institucional a los modelos de construcción del imaginario visual y de producción de significado que se dan en el contexto cultural. Cuando dicha mediación invita al análisis y a la crítica desde dentro del propio sistema, crea la impresión de que la institución ha resultado ser complaciente y dispuesta a someterse a un examen crítico, por tanto, es capaz de progresar, de reavivar el diálogo con la contemporaneidad y de cuestionar los modelos establecidos. En el escenario norteamericano y europeo, el contexto específico del arte ha resultado atractivo para los museos que han buscado progresivamente el visto bueno del ámbito de la crítica cultural. La institución se vuelve, de este modo, acomodada y acoge con agrado al artista o crítico comprometido en la deconstrucción de un contexto institucional como parte del programa. Incluso diría que no hay programa de interés que no incluya prácticas artísticas en torno a la crítica institucional. Resulta paradójico y contradictorio que la crítica a la institución sea enmarcada por el propio organismo. Aun así, es necesaria la crítica institucional, del mismo modo que es clave situarse críticamente ante el discurso dominante. 
Por otra parte, el museo es el que valida y legitima como arte cualquier producción cultural. Esta mediación se conforma como una estructura institucionalizada para la transferencia del conocimiento. Sin embargo, datos estadísticos de asociaciones de mujeres artistas siguen evidenciando, en el siglo XXI, las diferencias que siguen existiendo entre géneros en los programas expositivos y en las adquisiciones de obras para las colecciones de los museos. Del mismo modo, advierten sobre el número de obras de autoría femenina confinadas a las áreas de depósito y sepultadas de por vida.

Maura Reilly, comisaria, crítica de arte, directora de la National Academy of Desing de Nueva York fue la comisaria fundadora del Elizabeth A. Sackler Center For Feminist Art en el Brooklyn Museum, el primer espacio del mundo dedicado exclusivamente al arte feminista. Reilly analiza la noción clásica de museo como una institución pública dentro del sistema del arte encargada de clasificar, jerarquizar, catalogar y organizar desde narraciones lineales y hegemónicas que privilegian la primacía de la raza blanca y lo masculino. En el ensayo publicado en 2017 en Artnews, bajo la pregunta: What is curatorial activism? (incluido en su último libro: Curatorial Activism: Towards an Ethics of Curating $1^{9}$ señala la importancia de promover un tipo de comisariado focalizado en organizar exposiciones de arte que rescaten o den cuerpo visible y lugar en la historia a todos esas minorías que han sido silenciadas o excluidas de las narrativas maestras del arte: mujeres, artistas de color, artistas que no son blancos, todos aquellos no euroamericanos, artistas queer o todas aquellas identidades minoritarias que no son tenidas en cuenta por cuestiones relativas a su sexualidad, etc. El ensayo de Reilly recorre aquellas propuestas expositivas que, aun siendo consideradas controvertidas por contrahegemónicas, han funcionado como correctivos comisariales dado que recuperan muchos años de injusta omisión a las artistas mujeres, al mismo tiempo que abren nuevas modos de entender el comisariado. Reilly insiste en posicionarse como resistencia frente al heterocentrismo y la lesbo-homofobia y nos alerta señalando que estos no son problemas del pasado. Informa del escalofriante panorama que muestran las estadísticas actuales en las que se siguen obviando nombres por discriminación sexista y racista, a pesar de todas estas décadas de activismo y análisis acerca de lo subalterno, las teorías poscoloniales, los feminismos, la raza, las minorías LGBTQ, etc., En el sistema del arte todo está afectado: desde las galerías, los precios de las obras en el mercado secundario - subastas, la repercusión en prensa, la adquisición de obras para colecciones, los programas expositivos de los museos. Son reveladores los datos que Reilly nos ofrece al revisar algunos de los grandes eventos expositivos, como las bienales o los museos mediáticos que están en los circuitos del turismo cultural, en clave estadística. La mayoría de las exhibiciones continúan en la 
dinámica de presentar artistas blancos, euroamericanos, heterosexuales y sobre todo hombres. El mundo del arte continúa en el siglo XXI esencialmente sexista (Reilly, 2017).

Por mucho que se postule que provocar discursos de resistencia a las narrativas hegemónicas conformadas a partir de un poliédrico crisol de minorías de raza es ignorar a la raza blanca europoide o caucasoide y su gran variedad de fenotipos como identidad genética. Lo cierto es que esas minorías consideradas alteridad, en ocasiones, han sido manipuladas en el espacio museístico para provocar la enfatización de lo occidental-leucodermo y lo masculino. En cualquier caso, habría que huir de estrategias imprudentes y poco serias como la llevada a cabo en la Bienal de Whitney de 1993. Una manipulación política, a modo de performance, para demostrar que el arte norteamericano no es de predomino blanco. La estrategia consistió en incluir en las insignias o pegatinas, que te colocan en la entrada del museo, frases diseñadas por un artista de origen latino, Daniel Martínez, en las que se podía leer: «l Can’†», «lmagine Ever», «Wanting To», «Be White» $y$ «l can't imagine ever wanting to be White». Si con ese gesto el museo se reconocía sensible a lo multirracial al mismo tiempo anulaba un juicio crítico razonado al ignorar un grupo biológico protoeuropeo.

La reapertura de la Tate Modern en Londres, en 2016, ejemplariza el sexismo habitual de los museos: de los trescientos artistas representados en la colección permanente, menos de un tercio eran mujeres y de ese tercio muy pocas no eran blancas. En la colección del Centro Pompidou, que abarca obras desde 1900 hasta la época actual, menos del 10\% de dichas obras son de mujeres y menos del 10\% son de artistas que no son blancos. En el Metropolitan Museum of Art, en el apartado de arte moderno, el número de obras de mujeres es inferior al $4 \%$, no habiendo artistas no blancos. La edición de Venecia de 2017, comisariada por Christine Macel bajo el título Viva Arte Viva, estuvo protagonizada en un $61 \%$ por artistas europeos y norteamericanos, la cuota ocupada por artistas feministas fue del $35 \%$. En el programa se incluyó el activismo africano como el del grupo Black Lives Matter, con 120 artistas de los cuales solo una era mujer. En relación a las galerías de arte, Reilly menciona estadísticas estudiadas en el año 2015 en base a los datos que las propias galerías de arte aportan en su página web, como por ejemplo la lista de artistas que representan (Reilly, 2017). Los porcentajes del 2015 son prácticamente similares en el 2018. Entre las galerías estudiadas, reconocidas internacionalmente, mencionaremos a: Hauser Wirth \& Schimmel en Los Ángeles, que cuenta con un 32 \% de mujeres artistas en 2015 y $29 \%$ en 2018 , quizá la que más se aproxima a la paridad; o las galerías neoyorquinas como la Pace Gallery que sólo cuenta con un 14 \% en 2015 y un 10\% en 2018; la Gagosian con un $15 \%$ en 2015 y un $14 \%$ en 2018 y David Zwirner con un $28 \%$ en 2015 y un $19 \%$ en $2018^{10}$.

10 Los porcentajes aportados por Reilly son del año 2015. Véase en Reilly, Maura (11/07/17) "What is curatorial activism?» Artnews.com y en Reilly, Maura (2017). Notes from the inside: building a center for feminist art en https://w.w.w.maurareilly.com 


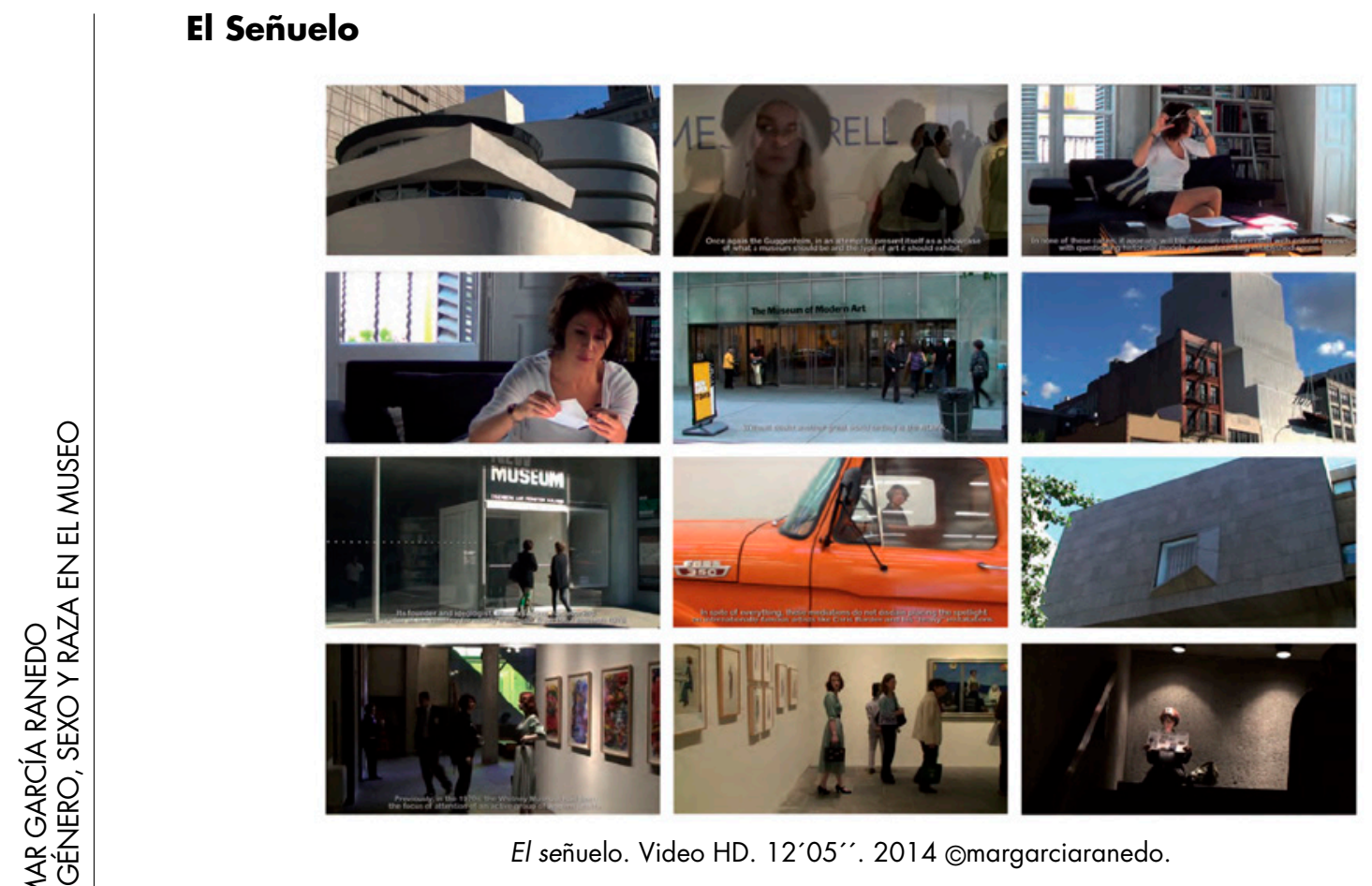

El canon tradicionalmente masculinista del museo es el argumento de la videocreación titulada El señuelo que cierra este artículo, creada por mí en el año 2014 ${ }^{11}$. En el vídeo, en dos tiempos paralelos, ocurren dos acciones: una consistente en una insurrección; en la que introduzco mi propio pelo en un sobre para depositarlo en cuatro museos. Una acción que también puede ser interpretada como la injerencia de particularismos en un órgano preponderante de la cultura global y que, por tanto, es siempre indiferente, dada su posición de organizador, clasificador y seleccionador, a diferentes estratos comportamentales y creativos. El museo aún hoy es exclusivo, su capacitación para ser el órgano mediador entre el discurso y el espectador hace de este un contenedor selectivo no inclusivo. La otra acción propone un paseo, un recorrido crítico narrado por una voz en off, en la que una serie de acontecimientos puntuales ponen de manifiesto ciertos particularismos discriminatorios o segregacionistas acontecidos en los emblemáticos museos de New York: Guggenheim, MoMA, Whitney y New Museum. 
El punto de partida del recorrido comienza en el museo Guggenheim. Su rotonda en espiral aparece tapiada por una envolvente tela con la intención de focalizar la mirada del espectador en la exposición del conocido artista norteamericano James Turrel. Una vez más el Guggenheim, en su intento de presentarse como referencia de lo que un museo debe ser y el tipo de arte que debe mostrar, se embarca en una megalómana concepción del formato expositivo y en un modelo de arte pretendidamente globalizado y universal. Del mismo modo que en sus otras sedes franquicias, en Nueva York también la plusvalía se mide en colas de visitantes a la espera de entrar en algunas de sus grandilocuentes instalaciones. De este modo, el museo genera capital vinculado al turismo cultural. En la década de los noventa, el Guggenheim planteó una nueva sede en Nueva York diseñada por Frank Gehry que posteriormente desestimó, aunque el arquitecto y la Fundación planean una sede faraónica para Abu Dhabi.

El recorrido videográfico continúa en el Whitney Museum. En el año 1987, las Guerrilla Girls pusieron de manifiesto la falta de representación de las mujeres artistas de raza negra en la historia del Whitney con una exposición externa al ámbito museístico que titularon Guerrilla Girls review the Whitney. Anteriormente, en la década de los setenta, el Whitney Museum había sido foco de atención para un activo grupo de mujeres artistas que llevaron a cabo un decidido esfuerzo para luchar contra la segregación en el espacio del museo por razones de género y raza. Este colectivo conocido como The Ad Hoc Women's Art Committee, formado por artistas como Brenda Miller, Faith Ringgold, Poppy Johnson y críticas como Lucy Lippard protestó contra el predominio del género masculino en las listas de exposiciones que el Whitney organizaba anualmente. Para la exposición anual de 1969 fueron invitados 151 artistas de entre los cuales sólo 8 eran mujeres. En la inauguración, y a modo de protesta, estas artistas depositaron huevos marcados con un porcentaje del $50 \%$, al tiempo que proyectaron en las paredes exteriores del Whitney diapositivas de obras de todas las artistas que no habían sido tenidas en cuenta siendo excluidas por cuestiones de sexo y raza. El museo reaccionó al accionismo de Ad Hoc Women's Art Committee incluyendo el 23 $\%$ de artistas mujeres en la muestra anual de escultura organizada al año siguiente, en 1970. Si bien, era más del doble del año anterior, no alcanzó el 50\% que Lippard y su grupo de artistas activistas demandaban. Lippard denunció la situación de desigualdad al Art Workers' Coalition, AWC: «Los museos deben alentar a las artistas mujeres para vencer centurias de daño hecho en la imagen de la mujer como artista estableciendo una representación equitativa de los sexos en las exhibiciones y colecciones» (Butler, 2012: 51).

La nueva sede del Whitney Museum, inaugurada, en Nueva York en el año 2015, con una exposición temporal titulada "America is hard to see», con obras de su propia 
colección que abarcaban todo el siglo XX hasta la actualidad, el $69 \%$ eran artistas hombres y el total de artistas blancos fue de un $77 \%$, es decir, el $11 \%$ eran mujeres blancas y el $20 \%$ artistas hombres no blancos. La Bienal de Whitney del año 2014 fue criticada debido al racismo y sexismo que mostraron sus propuestas. Fue motivo de quejas por el grupo de artistas activistas conocido como «cliterati» denunciando la falta de mujeres en la exhibición: de los 103 artistas, solo 37 eran mujeres. Durante la inauguración dispersaron flyers por el suelo en los que se leía: «La inclusión de gente de color en los espacios expositivos para blancos no los hace menos coloniales o más radicales, eso es la retórica del multiculturalismo imperialista, una cagada de teoría. La Bienal de Whitney de 2014 ni siquiera se molestó en incluir a más artistas de color. Fue un gesto meramente retórico» ${ }^{12}$. Afortunadamente, las protestas surtieron efecto y en 2017, 28 de los 63 artistas eran mujeres y el porcentaje de artistas blancos y no blancos se aproximó bastante.

Otro de los grandes escenarios mundiales es, sin duda, el MoMA. Las relaciones de poder sociales y económicas que gobiernan el museo provocan que sea difícil definir si verdaderamente los planteamientos expositivos que ellos exhiben se corresponden con la realidad del arte contemporáneo. La reforma del año 2004 de este espacio pretendió, fundamentalmente, reavivar un museo anclado en una modernidad ya superada con la pérdida de los grandes relatos y la idea desvanecida de la globalización y los universalismos. Con la nueva ampliación, el MoMA determinó alejarse de etiquetas que lo vincularan a lo que Guy Debord (1967) denominó la cultura del espectáculo, tan arraigada en ciertas prácticas artísticas de la década de los ochenta. En el año 2005, bajo la propuesta de Sarah Peters, el museo decidió revisar el canon masculinista que predominó en las colecciones y los programas expositivos a lo largo de su trayectoria. Para lo que ideó llevar a cabo el Woman Project. Con la intención de reactivar el diálogo y cubrir aquellos vacíos históricos en los que el museo no había tenido en cuenta los discursos identitarios en relación al género y la raza, se crearon espacios de debate, simposios, talleres pedagógicos y didácticos, publicaciones, mayor número de exposiciones de artistas mujeres así como un mayor número de adquisición de obras de mujeres. Al siguiente año, en 2006, se conformó The feminist art Project, TFAP, siguiendo la línea del Elizabeth A. Sackler Center for Feminist Art of the Brooklyn Museum junto con algunas de las artistas feministas emblemáticas de la década de los setenta: Arlene Raven, Judy Chicago, Dena Muller, Judy Brodsky, Ferris Olin y Susan Fisher Sterling ${ }^{13}$. (Reilly, 2016)

12 https://hyperallergic.com/127047/feminist-protest-disrupts-the-whitney-biennial/ (Consultado el 15/2/2018)

13 Ver artículo publicado en ONCURATING.org (2016) titulado: Living the revolution: A dialogue between Maura Reilly y Lara Perry. Traducido como: «Viviendo la revolución: Un diálogo entre Maura Reilly y Lara Perry». 
En 2015, Maura Reilly publica en Artnews el ensayo: Taking the measure of sexism: facts, figures and fixes ${ }^{14}$ en el que hizo evidente la disparidad que existe en relación al género respecto a artistas mujeres incluidas en los programas expositivos así como al número de obras de artistas mujeres que forman parte de la colección permanente del MoMA. Después de diez años, si bien se han modificado, los porcentajes no llegan a mostrar paridad ni por cuestiones de sexo como tampoco de raza. En 2004, el 4\% de artistas en exhibición eran mujeres y un porcentaje menor eran artistas no blancos mientras que en 2016 se llegó al $21 \%$ para las mujeres y $14 \%$ el de artistas no blancos.

El último espacio museístico abordado en el video es el New Museum. Reinaugurado en el año 2007 en un edificio diseñado por la pareja de arquitectos japoneses que conforman la firma SANAA ${ }^{15}$, pretende ser el templo del arte contemporáneo en la actualidad. Su fundadora e ideóloga, Marcia Tucker, había trabajado como conservadora en el Whitney durante casi una década, desde 1968 a 1976, y sufrió, en primera persona, todas las discriminaciones que iban adheridas al género:

Entonces, un día, mi colega Jim Monte me dijo que ganaba más que yo [...] El museo le había contratado a la vez que a mí, con el mismo cargo y las mismas funciones, pero le habían estado pagando unos dos mil dólares más al año. En 1969, dos mil dólares era casi una quinta parte de mi salario anual (Tucker, 2009: 126).

Ideó, por tanto, un espacio que posibilitara el diálogo con la contemporaneidad capaz de mostrar la realidad del arte sin prejuicios. En 1977 comienza a poner en pie todos los fundamentos que le habían motivado para crear un lugar alternativo que sirviese como catalizador entre la realidad colectiva, la construcción de la historia cultural y el museo. Marcia Tucker señala como «apartheid artístico» la marginación vivida por los artistas procedentes de culturas asiáticas, latinas y africanas. Las instituciones culturales han manejado estas minorías como lo distinto-otro que necesita ser exhibido en lugares y programas separados del relato oficial, y ser reducido a un esencialismo exclusivamente de tipo racial. El New Museum debía configurarse como una institución sin ánimo de lucro, fundamentada en la integración multirracial y multicultural tanto en sus programas expositivos como en su personal y sus órganos de gobierno.

Este centro se posiciona en la actualidad entre la sede tradicional y el espacio alternativo y se considera el primer espacio dedicado al arte contemporáneo establecido en

14 El artículo publicado en Artnews en 2017, Taking the measure of sexism: facts, figures and fixes, traducido como: «Tomando medida al sexismo: hechos, cifras y soluciones».

15 SANAA (Sejima + Nishizawa y Asociados) una firma de arquitectos con base en Tokio. 
la ciudad de Nueva York capaz de presentar la obra de artistas vivos que todavía no tienen una proyección pública amplia. A pesar de todo, estas mediaciones no desestiman poner el punto de mira en artistas conocidos internacionalmente como Chris Burden, no pudiendo finalmente escapar de la concepción del arte como dinamizador turístico y excedente económico y político.

La producción de conocimiento y la contribución a la historia del arte contemporáneo desde el museo se vuelve de este modo mediática, «interesada», creada conforme a una estructura hegemónica masculinizada que valoriza un cuerpo de normas inherentes de políticas basadas en el capital en un espacio de dominación cultural. El museo se enfrenta, de este modo, a la realidad cultural y artística practicada y vivida por medio de relaciones sociales y desempeños de género y minorías. El museo no es únicamente un almacén y expositor de subjetividades, es una conformación de conocimiento construida desde la investigación y la especificidad que siempre es avenencia. Cada museo busca una especificidad cultural que es diversa y no objetiva en relación a otros museos; lo que no es lícito es que en orden a esa subjetividad excluya espacios de expresión soberanos. El museo puede y debe ser subjetivo pero debe igualmente ser incluyente y reactivo a la sociedad para que el resultado de su investigación y, en ocasiones, de su colección sea un ancla entre lo real subjetivo y lo social objetivado. De no ser así, opera desde lo aprehendido sin ningún estímulo ni cuestionamiento, siendo un mediador alienado y alienante. Su operatividad como dispositivo y mecanismo de conocimiento y profundidad queda supeditada al orden de lo superficial/hegemónico. Su persistencia en las prácticas de predominio y preponderancia lo hacen dependiente y perverso puesto que alteran «interesadamente» el principio básico de servicio a la sociedad.

\section{Referencias y bibliografía}

BUtLER, Cornelia (2012). From conceptualism to feminism. Lucy Lippard's numbers shows 1969-74, Londres: Afterall Books.

CRIMP, Douglas (2005). Posiciones críticas. Ensayos sobre las políticas de arte y la identidad, Madrid: Akal.

DeBORD, Guy (1967). La Société du spectacle, París: Buchet-Chastel.

GerHARD, Jane (2013): The Dinner Party, Judy Chicago and the Power of popular feminism, 1970-2007, Georgia: University of Georgia Press, p.p. 185-187.

GIRLS, Gerrilla (1998). The Guerrilla Girls's bedside companion to the history of western art. New York, London: Penguin Books.

GuAsch, Ana María (ed.) (2000). Los manifiestos del arte posmoderno, Madrid: Akal. 
JONES, Amelia (1996). "The "sexual politics" of The Dinner Party», en CottINGHAM, Laura (ed.) (1996). Sexual Politics: Judy Chicago's Dinner Party in Feminist Art History, Los Ángeles: UCLA at the Armand Hammer Museum of Art and Culture Center y University of California Press.

Kelly, Mary (2001). "Contribuciones a una re-visión de la crítica moderna», en Walls, Brian (ed.) (2001). Arte después de la modernidad, Madrid: Akal.

KRAmer, Hilton (1980). «Art: Judy Chicago's Dinner Party comes to Brooklyn Museum», New York Times, October 17, en ReININK, Wessel y Jeroen Stumpel (Ed.) (1980). Memory \& Oblivion, Volume I, Springer-Science+Business Media, B.V.

LIPPARD, Lucy (1980). "Sweeping Exchanges: The contribution of feminist art of the 1970's», Art Journal 39, fall/winter, en LAUTER, Estella (1980). Women as Mythmakers, Bloomington: Indiana University Press.

_ (2004). Seis años: La desmaterialización del objeto artístico de 1966 a 1972, Madrid: Akal.

MoRAga, Cherríe y Gloria ANZALDÚA (1981). This bridge called my back: writings by radical women of color, Londres: Persephone Press.

MulveY, Laura (2001). «Placer visual y cine narrativo», en Walls, Brian (ed.) (2001). Arte después de la modernidad, Madrid: Akal.

NoCHIN, Linda (1971). «Why have there been no great women artists?», Art News, January p.p. 22-39; reimpresión en 1989, Women, art and power and other essays, New York: Thames and Hudson, p.p. 145-177.

Pollock, Griselda (2006). Differencing the canon, New York: Routledge.

_ (2010). Encuentros en el museo feminista virtual, Madrid: Ensayos Arte Cátedra.

Relly, Maura (2016). Living the revolution: A dialogue between Maura Reilly y Lara Perry, en http://www.maurareilly.com/essays.html, (Fecha de consulta: 15/02/2018).

_ (2017). What is curatorial activism?, en http://www.maurareilly.com/essays.html, (Fecha de consulta: 15/02/2018).

ReCKITt, Elena y Peggy Phelan (2001). Art and feminism, Nueva York, Phaidon Press Inc.

SMITH, Terry (2012). ¿Qué es el arte contemporáneo?, Buenos Aires: Siglo veintiuno editores. SolÁ, Miriam (2013). «Pre-textos, con-textos y textos», en SolÁ, Miriam y Elena URKO (coomps.) (2013). Transfeminismos, Orkoien, Navarra: Txalaparta.

TUCKER, Marcia (2008). 40 años en el arte neoyorquino, Madrid: Turner Noema.

Recibido el 1 de marzo de 2018

Aceptado el 16 de mayo de 2018 BIBLID [1 139-1219 (2018) 32: 43-61] 